\title{
Estudo com módulos auto-instrucionais como uma estratégia de ensino na disciplina de Enfermagem Neonatológica
}

\author{
Maria das Graças O. Fernandes ${ }^{1}$ \\ Vera Lúcia Barbosa ${ }^{2}$ \\ Masuco Naganuma ${ }^{2}$
}

Fernandes MGO, Barbosa VL, Naganuma M. Estudo com módulos auto-instrucionais como uma estratégia de ensino na disciplina de enfermagem neonatológica. Acta Paul Enferm 2005; 18(1):110-3.

\begin{abstract}
RESUMO: Nota prévia de um estudo quase experimento com delineamento de série de tempo, que procura descrever uma estratégia de ensino na disciplina de enfermagem neonatológica, com módulos auto-instrucionais de exame físico do Recém-Nascido a Termo (RNT). O locus desta pesquisa é uma Universidade privada do Estado de São Paulo. Os participantes utilizarão módulos auto-instrucionais em forma de $C D$-ROM para aprendizagem do exame físico do RNT. Este estudo trará contribuição aos docentes de enfermagem e aos alunos quanto a auto aprendizagem e desenvolvimento da habilidade na realização do exame físico do RNT.
\end{abstract}

Descritores: Enfermagem neonatal; Exame físico; Recém-nascido; Modelos educacionais

- Artigo recebido em 02/04/03 e aprovado em 18/02/04

\section{INTRODUÇÃO}

Em nossa experiência como docentes na disciplina de enfermagem neonatológica, percebemos a dificuldade do aluno associar o conteúdo teórico recebido em sala de aula com a prática. A preocupação com essa dicotomia nos impulsionou a realizar este estudo, buscando direcionar o processo ensino-aprendizagem de forma mais eficiente para que o aluno execute a prática com segurança e fundamentado no conhecimento teórico científico.

O emprego de módulos autoinstrucionais como estratégia de ensino, visa estimular a auto-aprendizagem, gerando alunos conscientes da responsabilidade pelo seu desenvolvimento profissional e capazes de associar o conhecimento adquirido à tomada de decisão ao realizar um procedimento no campo clínico. Nesse contexto, há necessidade de que os docentes mudem de comportamento e possam refletir e até deixar de lado a tradição do processo educacional, permitindo que o aluno, de forma integrada, deixe de ser passivo para se tornar participante no processo ensino. No entanto, ressaltamos, que:

estudos em nível de micro-ensino são urgentes para diminuírem a lacuna entre o que deveria ser e o que é o processo de formação do enfermeiro $^{(1)}$.

Assim, o docente de enfermagem deve estar atento para pro- porcionar ao graduando uma experiência que contribua para a sua formação e que vá de encontro as suas necessidades de aprendizagem, tanto em sala de aula como no campo clínico $^{(2)}$. Pesquisa refere-se que os softwares educacionais, para determinados conteúdos "caem como uma luva" podendo ajudar e facilitar os processos de trabalhos, além de contribuírem nos processos cognitivos e motivacionais ${ }^{(3)}$. Portanto, este $C D$ - $R O M$ tem finalidades pedagógicas, e acreditamos que colabore no ensino do conteúdo do exame físico do RNT, como facilitador do processo ensinoaprendizagem na qualidade de ferramenta útil à docência em enfermagem neonatológica.

\footnotetext{
Mestranda em enfermagem pediátrica pela Universidade Federal de São Paulo UNIFESP/EPM. Professora da disciplina de enfermagem pediátrica e neonatológica, Processo de cuidar da Universidade Anhembi Morumbi. E-mail: enfgraca@ig.com.br

2 Professora Adjunto Doutor do Departamento de Enfermagem da Universidade Federal de São Paulo, UNIFESP/EPM.
} 
A utilização de Módulos autoinstrucionais permite aprender determinado conteúdo de forma independente, é um método que oferece alternativas para realizar a aprendizagem, sendo o aluno responsável pelo processo. A cada término do módulo, as avaliações são para verificar se atingiram os objetivos propostos pelo professor no assunto estudado. Os módulos auto-instrucionais, doravante, denominados módulos representam uma estratégia de ensino confiável que facilita o processo ensinoaprendizagem, desenvolvem habilidades (perceptivo e motora) e o auto-conhecimento usados na enfermagem desde o final da década de $1970^{(4-14)}$.

O fator motivador para trabalhar com essa estratégia baseou-se nas pesquisas realizadas com módulos auto-instrucionais em conteúdos diferentes que apresentaram resultados satisfatórios. Para elaborar o emprego dos módulos instrucionais, é necessário conhecer esse tipo de estratégia de ensino e identificar qual o melhor modo de utilizála, sendo preciso rever seu conceito. Esta estratégia de ensino acredita na capacidade do estudante para adquirir responsabilidade e confiança, fundamentando-se na aprendizagem para domínio de determinado assunto e no ensino baseado em competências ${ }^{(15-16)}$.

\section{Didática foi definida como}

estudo do conjunto de procedimento que visa orientar a aprendizagem do estudante mais eficientemente na aquisição de conhecimento, automatismo, atitudes e idéias

sendo os elementos da didática o aluno, o professor, o conteúdo, os objetivos e os métodos ${ }^{(17)}$. É através de módulos auto-instrucionais que são estudados a inter-relação destes elementos ${ }^{(16)}$. Os módulos auto instrucionais são instrumentos que levam o aluno a adquirir conhecimento por si mesmo, através da auto

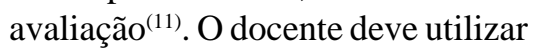
variadas estratégias de ensino, mas não pode esquecer o seu papel de facilitador do processo ensinoaprendizagem, sempre visando a qualidade do ensino. A utilização de módulos auto-instrucionais é uma estratégia simples e de baixo custo para ajudar o enfermeiro-docente a realizar o ensino-aprendizagem com mais eficiência e eficácia e contribuir para a formação do futuro profissional $^{(5)}$.

Este estudo tem como objetivos gerais: elaborar os módulos auto-instrucionais em forma de $C D$ $R O M$ e descrever o emprego da pesquisa quase experimento, aplicando módulos auto-instrucionais de exame físico RNT como estratégia de ensino, buscando verificar o desempenho na aprendizagem. Como pressuposto para este estudo, bom desempenho será considerada a nota igual ou maior que sete em uma prova escrita de conhecimento específico.

Hipótese de estudo: A utilização de módulos auto-instrucionais de exame físico de enfermagem do RNT como estratégia de ensino, levará o aluno a um bom desempenho dessa habilidade.

\section{MÉTODO}

Para verificar a hipótese do estudo está em andamento, uma pesquisa quase-experimental, com delineamento de série de tempo. Esse tipo de pesquisa é um simples experimento constituído por um grupo, previamente definido, para se verificar a influência da introdução de um estímulo $^{(18)}$ que será a utilização dos módulos auto-instrucionais, como estratégia de ensino no grupo determinado. A pesquisa quase-experimental com delineamento de série de tempo envolve a coleta de informações ao longo de um período de tempo (grupos de alunos antes e depois da utilização de módulos auto- instrucionais) quando ocorre a introdução de um estímulo, que vai permitir verificar a significância do estímulo introduzido ${ }^{(19)}$.

O estudo está acontecendo em uma Universidade privada na região do grande $\mathrm{ABC}$ no Estado de São Paulo. Fazem parte deste estudo alunos matriculados na graduação de enfermagem que estão cursando o $6^{\circ}$ semestre na disciplina de enfermagem neonatológica e que consentirem em participar do trabalho, assinando o Termo de Consentimento Livre e Esclarecido. Para verificação da hipótese serão utilizadas provas de conhecimentos específicos do exame físico do RNT antes e após emprego do $C D-R O M$ em forma de módulos auto-instrucionais.

\section{RESULTADOS PRELIMINARES}

Para a confecção dos módulos auto-instrucionais em forma de $C D$ $R O M$, o conteúdo sobre exame físico do RNT foi elaborado baseado na literatura norteadora do assunto, visando alunos da graduação de enfermagem. Foi organizada com base na proposta do plano Keller, estruturada no enfoque sistêmico ${ }^{(20)} \mathrm{e}$ fundamentada na teoria moderna da aprendizagem de Gagné ${ }^{(21)}$. Utilizou-se a adaptação do modelo de Price seguindo o modelo de Instrução Auxiliada pelo Computador (CAI) nas seguintes etapas: planejamento, desenvolvimento do $C D-R O M$ e avaliação $^{(22)}$. O primeiro momento do planejamento foi a seleção do tema, caracterização da população, estruturação do conteúdo como sistema instrucional, definição dos objetivos instrucionais, descrição dos recursos tecnológicos, materiais e humanos. A segunda etapa foi o desenvolvimento do $C D-R O M$, onde foram definidos: computação gráfica, edição do conteúdo de exame físico do RNT e diagrama de navegação. $\mathrm{Na}$ terceira etapa foi elaborada a forma de avaliação do conteúdo estudado por meio do CD-ROM. Antes 
de utilizar o $C D-R O M$ com alunos de graduação, o conteúdo do exame físico do RNT em forma de módulos auto-instrucionais passa no momento por uma avaliação feita por peritos da área de enfermagem neonatológica. Foram estabelecidos os seguintes critérios de inclusão dos avaliadores: enfermeiros docentes ou assistenciais ligadas à área de ensino, com no mínimo dois anos de experiência em neonatologia e ou pediatria no exercício pleno de suas atividades na área especifica e titulação mínima de especialista. Os peritos avaliam: objetividade do conteúdo; informações atualizadas; abrangência do tema; vocabulário empregado; forma, distribuição, apresentação do conteúdo (didática); descrição dos conceitos utilizados e critérios estabelecidos ou seja, insatisfatório, péssimo, regular, bom e excelente. Até o momento temos avaliação parcial dos peritos; após esta avaliação de todos os peritos do conteúdo do exame físico do RNT, será editado o $C D-R O M$ e aplicado e testado como estratégia de ensino para alunos da graduação de enfermagem na disciplina de enfermagem neonatológica.

\section{CONSIDERAÇÕES FINAIS}

Nota prévia de uma pesquisa em andamento sobre uma estratégia de ensino na disciplina de enfermagem neonatológica por meio de módulos auto-instrucionais em forma de CD-ROM do exame físico do RNT.

\section{REFERÊNCIAS}

1. Friedlander MR. O ensino dos procedimentos básicos no Laboratório de Enfermagem: comparação entre dois métodos de instrução. [tese doutorado]. São Paulo: Escola de Enfermagem da USP; 1984.
2. Oliveira I. Práticas e percepções de alunos de cinco cursos de graduação em enfermagem do Estado de São Paulo sobre alguns aspectos do ensino prático de enfermagem pediátrica realizada em hospital. [dissertação mestrado]. São Paulo: Departamento de Enfermagem da UNIFESP; 1989.

3. Juliani CMCM. Tecnologia educacional: produção e avaliação do site escala de pessoal de enfermagem [tese doutorado]. São Paulo: Escola de enfermagem da USP; 2003.

4. Friedlander MR. Ensino individualizado na enfermagem. Enferm Mod 1985; 3(4):22-8.

5. Friedlander MR, Tanaka CS, Siqueira PSF. Estímulos que favorecem o treinamento no Laboratório de Enfermagem: revisão de literatura. Rev Esc Enferm USP 1989; 23(2):115-25.

6. Friedlander MR, Tagawa MTC, Silveira C, Szobo MA. Estímulos que favorecem o treinamento no Laboratório de Enfermagem: opinião de professores e alunos. Rev Esc Enferm USP 1990; 24 (1):41-65

7. Friedlander MR. Vantagens do ensino no Laboratório de Enfermagem. Rev Esc Enferm USP 1994; 28(2):227-33.

8. Miyadahira AMK. Processo de ensinoaprendizagem de habilidades psicomotoras: análise da técnica de injeção intramuscular [tese doutorado]. São Paulo: Escola de Enfermagem da USP; 1990.

9. Nogueira MS. Aplicação de injetáveis via intramuscular: comparação entre dois métodos de ensino [tese doutorado]. São Paulo: Escola de Enfermagem de Ribeirão Preto/ USP;1995

10. Cassiani SHB. Um salto para o futuro no ensino da administração de medicamentos: desenvolvimento de um programa instrucional auxiliado pelo computador [tese livre-docência]. São Paulo: Escola de Enfermagem de Ribeirão Preto/ USP; 1998.
11. Coutinho RMC. Módulos auto-instrucionais no laboratório de enfermagem em centro cirúrgico [dissertação mestrado]. São Paulo: Departamento de Enfermagem da UNIFESP; 1999.

12. Paiva SS. Queimaduras: atendimento hospitalar ao paciente adulto na fase inicial da injúria - um software autoinstrucional [tese doutorado]. São Paulo: Escola de Enfermagem da USP; 2001.

13. Zem-Mascarenhas SH, Cassiani SHB. Desenvolvimento e avaliação de um software para o ensino de enfermagem pediátrica. Rev Lat Am Enferm 2001; 5(6):13-8.

14. Duran ECM, Cocco MIM. Software educativo sobre diabetes mellitus para profissionais de saúde: etapas de elaboração e desenvolvimento. Rev Lat Am Enferm 2003; 11(1):104-7.

15. Parra N. Ensino individualizado: programas e materiais. São Paulo: Saraiva; 1978.

16. Reis A, Joullié V. Didática geral através de módulos instrucionais. 7a ed. Petrópolis:Vozes;1988.

17. Nérici IG. Didática geral dinâmica. Rio de Janeiro: Fundo de Cultura; 1973.

18. Victoriano ERA, Garcia CC. Produzindo monografia: para trabalho de conclusão de curso - TCC. 3a ed. São Paulo: Publisher Brasil; 1995.

19. Polit DF, Hungler BP. Fundamentos da pesquisa em enfermagem. 3a ed. Porto Alegre: Artes Médicas; 1995.

20. Godoy SA. Recursos tecnológicos e ensino individualizado. In: Moreira, DA. Didática do ensino superior: técnica e tendências. São Paulo: Pioneira; 1997. cap. 5, p. 101-13.

21. Gagné RM. Princípios essenciais da aprendizagem para o ensino. Trad. de Rute Vivian Ângelo. Porto Alegre: Globo; 1980.

22. Price RV. Computer-aided instruction: a guide for authors. Pacific Grove: Brooks Cole; 1991. 
Fernandes MGO, Barbosa VL, Naganuma M. [Study with selfinstructional modules as a teaching strategy in the neonatological nursing.] Acta Paul Enferm 2005; 18(1):110-3.

ABSTRACT: This is a previous note of an almost experimental study with time series aiming teaching strategy in neonatal nursing discipline with self-instructional modules of physical examination in Full-Term Newborns Infant (TNBI). The site of this research wil be a private University in the State of São Paulo. The particicipants will use CD-ROM self-instructional modules to learn how to perform physical examination in TNBI. This study will bring much contribution to the nursing academic staff regarding their self-learning and development in the ability to perform physical examination in TNBI.

Descriptors: Neonatal nursing; Physical examination; Infant, newborn; Models, educational
Fernandes MGO, Barbosa VL, Naganuma M. [Estudio con módulos auto-instruccionales como una estrategia de enseñanza en la disciplina de enfermería neonatológica.] Acta Paul Enferm 2005; 18(1):110-3.

RESUMEN:Nota previa de un estudio casi experimental con delineamiento de serie de tiempo que tiene como objetivo la estrategia de enseñanza de enfermería neonatal, con módulos auto-instruccionales en al examen físico de los Recién Nacidos a Término (RNT). El locus de esta pesquisa será en una Universidad privada del Estado de São Paulo. Los participantes irán utilizar módulos auto-instruccionales en forma de CD-ROM para el aprendizaje del examen físico del RNT. Esse estudio hará una contribuición a los docentes de enfermería y a los alumnos cuanto al auto-aprendizaje y desarrollo de la habilidad en I realización del examen físico del RNT.

Descriptores: Enfermería neonatal; Examen físico; Recién nacido; Modelos educacionales 